\title{
Rapidity and reliability of gonococcal identification by coagglutination after culture on modified New York City medium
}

\author{
H YOUNG* AND A MCMILLAN†
}

From the *Department of Bacteriology, Edinburgh University Medical School and Royal Infirmary; and the +University Department of Genitourinary Medicine, Royal Infirmary, Edinburgh

SUMMARY The combination of culture on modified New York City (MNYC) medium and identification of neisserial isolates by the Phadebact ${ }^{\circledR}$ gonococcus test was evaluated in routine laboratory practice. The sensitivity of coagglutination was $96 \cdot 7 \%$ (318/329 isolates) and that of immunofluorescence (IF) $97 \cdot 9 \%$ (322/329 isolates); the specificity of both methods was $96 \cdot 8 \%$ $(120 / 124$ isolates). Of the 329 gonococcal isolates, $286(86 \cdot 9 \%)$ could be tested by coagglutination and $309(93.9 \%)$ by IF after only 24 hours' incubation. Identification by coagglutination from primary cultures on MNYC medium is considered to be very rapid, simple, and efficient for the cultural diagnosis of anogenital gonorrhoea in women and urethral gonorrhoea in men. Because of the high prevalence of meningococci in the pharynx and their not infrequent occurrence in the anorectum of homosexual men the identity of isolates from these sites is best confirmed by sugar utilisation tests.

\section{Introduction}

The Phadebact ${ }^{\boxplus}$ gonococcus test (Pharmacia Diagnostics AB, Uppsala, Sweden) is a rapid slide test for the immunological identification of Neisseria gonorrhoeae. The test depends on the principle of coagglutination. ${ }^{1}$ An early report from the United Kingdom $^{2}$ of gonococcal identification by coagglutination was critical of the specificity of the method. More recent reports from the United States, ${ }^{3-5}$ Australia, ${ }^{6}$ and Switzerland ${ }^{7}$ suggest that coagglutination can provide rapid and accurate immunological confirmation of $N$ gonorrhoeae.

Rapid cultural diagnosis of gonorrhoea, particularly in women, is of prime importance in the control of infection within the community. Modified New York City (MNYC) medium promotes rapid gonococcal growth and enables biochemical characterisation to be carried out directly from the primary isolation plate, providing a rapid and economical system of identification. ${ }^{8}$ An improve-

Address for reprints: Dr $\mathbf{H}$ Young, Department of Bacteriology, Edinburgh University Medical School and Royal Infirmary, Teviot Place, Edinburgh EH8 9AG

Accepted for publication 26 October 1981 ment in the cultural diagnosis of gonorrhoea associated with the use of MNYC medium has been reported in the United Kingdom, ${ }^{9}$ Norway, ${ }^{10}$ and New Zealand. ${ }^{11}$ The aim of the present study was to evaluate the combination of culture on MNYC medium with identification by the Phadebact $^{\circledR}$ gonococcus test in routine laboratory practice.

\section{Patients and methods}

\section{STUDY POPULATION}

The patients in this study attended the department of genitourinary medicine at the Edinburgh Royal Infimary on a Monday, Tuesday, or Wednesday morning between November 1980 and May 1981 . Investigations to detect or exclude gonococcal infection were as described. ${ }^{9}$ All isolates of oxidasepositive Gram-negative diplococci were tested for penicillinase production and identified by the rapid carbohydrate utilisation test and by delayed immunofluorescence with Difco fluorescein-labelled antigonococcal conjugate. ${ }^{8}$ The coagglutination test was performed as described below. Cultures giving negative results were re-examined after incubation for 48 hours. 


\section{COAGGLUTINATION TEST}

\section{Lyophilised reagents}

Commercially available test kits (Phadebact ${ }^{\circledR}$ gonococcus test) were provided by Pharmacia Diagnostics AB, Sweden. A heavy suspension of each culture to be tested was made by removing the growth from the culture plate with a cotton-tipped swab and emulsifying it in $0.2 \mathrm{ml}$ distilled water in a tube. The tube was covered and the suspension heated in a boiling water-bath for five minutes. After cooling to room temperature $40-\mu \mathrm{l}$ aliquots of suspension were mixed with an equal volume of test reagent (rabbit antigonococcal antiserum bound to dead staphylococci) and control reagent (non-immune rabbit serum bound to dead staphylococci) on a clear glass slide: three tests were carried out on a single slide $(10.7 \mathrm{~cm} \times 5.0 \mathrm{~cm})$ with six clearly defined areas. The slide was rocked gently for two to three minutes by tilting to an angle of $45^{\circ}$ every two to three seconds and the results read against a dark background using indirect light.

A markedly stronger reaction with the gonococcal reagent compared with the control reagent constituted a positive result. No reaction with the gonococcal reagent irrespective of any reaction in the control reagent was considered a negative result. A reaction of equal strength in both the gonococcal and the control reagents was classed as non-interpretable.

Isolates giving false-positive and false-negative results by sugar utilisation reactions were retested the next day as a 24-hour subculture. A suspension of growth from each subculture was also made in skimmed milk $(10 \%)$ and stored frozen at $-40^{\circ} \mathrm{C}$.

\section{Liquid reagents coloured with methylene blue}

On completion of the trial isolates were reconstituted from the skimmed milk and tested with the new liquid reagents and with the conventional lyophilised reagents.
ANALYSIS OF RESULTS

Coagglutination and IF test results were correlated with sugar utilisation reactions. As the aim was tộ. make an 'in use' evaluation, only gonococcal isolates. giving clear-cut positive results with IF or coagglutin ation or both were scored positive; borderline reactions were scored negative. Conversely border $\frac{\bar{\sigma}}{\bar{\omega}}$ line reactions with non-gonococcal neisseriae wer⿸户巳 scored positive.

The sensitivity of coagglutination and IF method§ was defined as the percentage of gonococcal isolates confirmed by carbohydrate utilisation tests that were IF-positive or coagglutination-positive, while specificity was defined as the percentage of non $\frac{\widehat{S}}{\omega}$ gonococcal isolates confirmed by carbohydrate utilisation testing that was coagglutination-negative or IF-negative.

The significance of differences in the results with coagglutination and IF methods was determined by dividing the difference between the two totals to be compared by the standard error of the difference: a value equal to or greater than 1.96 indicates significant difference at the $5 \%$ level.

\section{Results}

SPECIFICITY AND SENSITIVITY

There were 140 men (mean age $29 \cdot 7$ years) and 1180 women (mean age $22 \cdot 8$ years) from whom oxidase positive Gram-negative diplococci (GNDC) were isolated: 189 isolates of GNDC were obtained from $\overrightarrow{5}$ 156 routine examinations of men and 264 isolates of GNDC from 131 routine examinations of women The coagglutination, immunofluorescence (IF), and sugar utilisation results for the 453 isolates are shownos in table $I$.

The sensitivity of the coagglutination test was $96 \cdot 7 \%(318 / 329$ isolates) and that of the IF testo $97 \cdot 9 \%(322 / 329$ isolates $)$. This difference is not

TABLE I Results of coagglutination, immunofluorescence, and sugar utilisation tests applied to 453 neisserial isolates.

\begin{tabular}{|c|c|c|c|c|c|c|c|c|}
\hline \multirow[b]{2}{*}{ Site } & \multirow{2}{*}{$\begin{array}{l}\text { No of cultures yielding } \\
\text { oxidase-positive GNDC }\end{array}$} & \multicolumn{3}{|c|}{ Identification by sugar utilisation } & \multicolumn{2}{|c|}{ Immunofluorescence } & \multicolumn{2}{|c|}{ Coagglutination } \\
\hline & & $N$ gonorrhoeae & $\bar{N} \overline{\text { meningitidis }}$ & N lactamica & Positive & Negative & Positive & Negative \\
\hline Urethra & 170 & 170 & & & \multirow{2}{*}{$\begin{array}{r}166 \\
86\end{array}$} & 4 & 165 & 5 \\
\hline \multirow[t]{2}{*}{ Cervix } & 89 & 88 & & & & 2 & 85 & 3 \\
\hline & & & \multicolumn{2}{|l|}{1} & 0 & 1 & 0 & 1 \\
\hline Rectum & 49 & 48 & \multirow{2}{*}{\multicolumn{2}{|c|}{1}} & 47 & 1 & 45 & 3 \\
\hline \multirow{5}{*}{$\begin{array}{l}\text { Bartholin's } \\
\text { gland } \\
\text { Throat }\end{array}$} & & & & & 0 & 1 & 0 & 1 \\
\hline & 8 & 8 & \multirow{4}{*}{115} & & 8 & 0 & 8 & 0 \\
\hline & 137 & 15 & & & 15 & 0 & 15 & 0 \\
\hline & & & & & 3 & 112 & 4 & 111 \\
\hline & & & & 7 & 1 & 6 & 0 & $7 *$ \\
\hline Total & 453 & 329 & 117 & 7 & 326 & 127 & 322 & 131 \\
\hline
\end{tabular}

GNDC = Gram-negative diplococci
*Includes one non-interpretable resul 
significant at the $5 \%$ level. The specificity of both coagglutination and IF was $96 \cdot 8 \% \quad(120 / 124$ isolates). Four isolates of $\beta$-lactamase-producing gonococci gave positive results by both methods.

\section{RAPIDITY OF DIAGNOSIS}

Table II shows the rapidity of gonococcal identification by coagglutination and IF methods. The difference in the total number of isolates from all anatomical sites tested at 24 hours by each method was not significant at the $5 \%$ level.

Of the 115 meningococci isolated from throat cultures in men and women $96(83.5 \%)$ were tested at 24 hours by coagglutination and $113(98 \cdot 3 \%)$ by IF.

\section{FALSE-POSITIVE AND FALSE-NEGATIVE COAGGLUTINATION RESULTS}

Seven isolates of gonococci (four from men and three from women) gave false-negative results when first tested. Only six of these isolates were available for retesting at the end of the trial and all gave a positive result. With the conventional freeze-dried reagents at least two minutes were required to produce coagglutination whereas this was clearly observable within $\mathbf{3 0}$ seconds with the liquid reagents containing methylene blue.

All four isolates of $N$ meningiditis (two serogroup $B$ and two non-groupable) that gave positive reactions initially gave negative results on retesting at the end of the survey.

\section{Discussion}

Our results indicate clearly that the overall sensitivity and specificity of coagglutination and IF are comparable. Similar results were obtained by Rufli $^{7}$ and by Johnston, ${ }^{12}$ who prepared her own coagglutination reagents. We found the coagglutination method was simple to perform, easy to interpret, and does not require expensive immunofluorescence equipment.
These advantages are of less benefit in laboratories with facilities for fluorescence microscopy and adequately staffed with skilled technologists. The cost of performing a coaggluíination test with commercial reagents is approximately $£ 0.50$ when a $40-\mu l$ volume is used compared with a cost of $£ 0 \cdot 10$ for an IF test with a 10- $\mu$ l unit volume. If coagglutination can be performed equally well with a 10- $\mu$ l volume, however, as reported by Futrovsky et $a l^{13}$ this would reduce the cost per test to approximately $£ 0 \cdot 125$.

Both coagglutination and IF methods have the same disadvantages: the failure to detect a small proportion of gonococcal isolates $(2-3 \%)$ and the failure to obtain a clear-cut negative reaction with a small proportion of non-gonococcal neisseriae. These problems can be minimised by using a carefully absorbed antigonococcal serum known to be reactive in the coagglutination test with local gonococcal strains. ${ }^{12}$ Despite these limitations immunological identification is a very useful adjunct in the diagnostic laboratory. Confirmation of the identity of gonococci is obtained rapidly and with very small amounts of bacterial growth.

Provided that unequivocal results are obtained the specificity and sensitivity of immunological methods are such that further identification of urethral isolates from men and anogenital isolates from women is unnecessary provided that colonial morphology is typical and correlates with immunological findings. Whenever medico-legal proceedings may be involved full identification including sugar utilisation tests must be performed. Because meningococci are isolated from the throat much more often than gonococci, the small number of false-positive reactions that may occur decreases the utility of immunological identification of pharyngeal isolates.

Although there are several reports of the isolation of $N$ meningitidis from anogenital sites, ${ }^{14-16}$ when the

TABLE II Proportion of gonococcal isolates from various anatomical sites tested by immunofluorescence and coagglutination after incubation of primary cultures for 24 hours

\begin{tabular}{|c|c|c|c|}
\hline \multirow[b]{2}{*}{ Site } & \multirow[b]{2}{*}{ No of isolates } & \multicolumn{2}{|c|}{ No $(\%)$ of isolates tested at 24 hours by: } \\
\hline & & Immunofluorescence & Coagglutination \\
\hline Male urethra & 106 & $104(98 \cdot 1)$ & $104(98 \cdot 1)$ \\
\hline Cervix & 88 & $85(96 \cdot 6)$ & $79(89 \cdot 8)$ \\
\hline Male rectum & 4 & $4^{*}$ & $4^{*}$ \\
\hline Female rectum & 44 & $40(90 \cdot 9)$ & $37(84 \cdot 1)$ \\
\hline Bartholin's gland & 8 & $8 *$ & $6^{*}$ \\
\hline Female throat & 8 & $7 *$ & $5^{*}$ \\
\hline Total & 329 & $309(93 \cdot 9)$ & $286(86 \cdot 9)$ \\
\hline
\end{tabular}

*Percentages not given if less than 10 observations 
incidence of such occurrences in relation to laboratory practice are considered the data must be interpreted carefully with reference to the particular patient groups concerned. In this study neisseriae were isolated from an anogenital site in $\mathbf{9 5}$ women (urethra, cervix, and rectum were tested routinely), but only one isolate $(1 \%)$ proved to be a meningococcus. Since $3 \cdot 2 \%$ of meningococcal isolates gave a false-positive reaction on initial testing a falsepositive coagglutination result with a meningococcus from an anogenital site in a female patient is unlikely to occur in more than 1 in $\mathbf{3 0 0 0}$ patients from whom anogenital neisseriae are isolated; this could be less than 1 in 30000 patients investigated. We consider identification by immunological methods acceptable at the above levels particularly when it is borne in mind that colonial morphology may alert a trained observer to a misdiagnosis.

In homosexual men non-gonococcal neisseriae appear to be isolated from the anorectum more frequently. In this trial one of five neisserial isolates from the anorectum of men was a meningococcus. In an earlier study ${ }^{17}$ we isolated $N$ meningitidis from the anorectum of four $(5 \cdot 7 \%)$ of 70 homosexual men. We maintain our earlier view that it is important to confirm, preferably by a rapid carbohydrate utilisation test, the identity of neisseriae isolated from the anorectum of homosexual men. Tapsall and Cheng ${ }^{18}$ in a recent comparative study also noted that a rapid test using preformed enzymes was superior both in terms of accuracy and rapidity than two other systems, both of which required growth of the organism to produce a positive result.

The Phadebact ${ }^{\boxplus}$ gonococcus test in conjunction with culture on MNYC medium provides a very rapid and simple method of gonococcal identification for isolates from anogenital sites in women and from the urethra in men. Because technical manipulations are kept to a minimum the scheme lends itself well to large-scale screening for gonococcal infection among high-risk groups and quality control is easy for laboratories that isolate few gonococci.

We are grateful to our medical and non-medical colleagues in the departments of genitourinary medicine and bacteriology for their help with the collection and processing of specimens.
References

1. Danielsson D, Kronvall G. Slide agglutination method for the serological identification of Neisseria gonorrhoeae with antigonococcal antibodies adsorbed to protein A-containing. staphylocccci. Appl Microbiol 1974;27:368-74.

2. Barnham M, Glynn AA. Identification of clinical isolates of Neisseria gonorrhoeae by a coagglutination test. J Clin Pathoto 1978; 31: 189-93.

3. Hampton KD, Stallings RA, Wasilauskas BL. Comparison of a slide coagglutination technique with the Minitek system for confirmation of Neisseria gonorrhoeae. J Clin Microbiol $\bar{\Phi}$ 1979; 10:290-2.

4. Lewis JS, Martin JE. Evaluation of the Phadebact gonococcus? test, a coagglutination procedure for confirmation of Neisseria gonorrhoeae. J Clin Microbiol 1980; 11:153-6.

5. Lim DV, Wall T. Confirmatory identification of Neisseria gonorrhoeae by slide coagglutination. Can J Microbiol 1980; $\overrightarrow{-}$ 26:218-22.

6. Shanker S, Daley DA, Sorrell TC. A rapid slide coagglutination test-an alternative to the fluorescent antibody test for? the identification of Neisseria gonorrhoeae. J Clin Pathols $1981 ; 34: 420-3$

7. Rufli T. Identification of Neisseria gonorrhoeae in the routineiv venereological laboratory. Comparative study of coagglutination, direct immunofluorescence, and sugar fermentation reaction. Br J Vener Dis 1980;56: 144-7.

8. Young $\mathrm{H}$. Identification and penicillinase testing of Neisseria $\mathrm{O}$ gonorrhoeae from primary isolation cultures on Modified New York City medium. J Clin Microbiol 1978; 7:247-50.

9. Young H, Harris AB, Urquhart, D, Robertson DHH Screening by culture for the detection of gonorrhoea in women. Scot Med J 1979; 24:302-6.

10. Svarva PL, Maeland JA. Comparison of two selective media in $\overrightarrow{0}$ the cultural diagnosis of gonorrhoea. Acta Pathol Microbiop Scand (B) 1979;87:391-2.

11. Hookham AB. Thayer-Martin medium and modified New York City medium for the cultural diagnosis of gonorrhoea. BrO $J$ Vener Dis 1981;57:213.

12. Johnston NA. Evaluation of the coagglutination test for the identification of Neisseria gonorrhoeae in primary cultures. Bro $J$ Vener Dis 1981;57:315-9.

13. Futrovsky SL, Gaydos CA, Keiser J. Comparison of the Phadebact Gonococcus Test with the rapid fermentation method. J Clin Microbiol 1981;14:89-93.

14. Willmott FE. Meningococcal salpingitis. Br J Vener Dis $1976 ;$ 52:182-3. 15. Miller MA, Millikin P, Griffin PS. Neisseria meningitidis
urethritis. A case report. JAMA 1979;242:1656-7.

16. Givan KF, Thomas BW, Johnston AC. Isolation of Neisseria meningitidis from the urethra, cervix and anal canal. Further observations. Br J Vener Dis 1977; 53:109-12.

17. McMillan A, Young H. Gonorrhoea in the homosexual man: frequency of infection by culture site. Sex Transm Dis 1978 5: $146-50$.

18. Tapsall JW, Cheng JK. Rapid identification of pathogenic species of Neisseria by carbohydrate degradation tests: importance of glucose in media used for preparation of inocula. Br J Vener Dis 1981;57:249-52. 